\title{
Massive companions of binary systems
}

\author{
D. Jableka ${ }^{1}$, S. Zola ${ }^{1,2}$, B. Zakrzewski ${ }^{2}$, J. M. Kreiner ${ }^{2}$ and \\ W. Ogloza ${ }^{2}$ \\ ${ }^{1}$ Astronomical Observatory of the Jagiellonian University, \\ Orla 171, 30-244 Krakow, Poland \\ email: jableka@oa.uj.edu.pl \\ ${ }^{2}$ Mt. Suhora Observatory, Pedagogical University, \\ ul. Podchorazych 2, 30-084 Krakow, Poland \\ email: szola@oa.uj.edu.pl
}

\begin{abstract}
We examined the O-C diagrams of eclipsing binary systems and selected these exhibiting cyclic shape, either sinusoidal or quasi sinusoidal. Assuming these variations being due to the Light Time Travel effect (LTE), we estimated the parameters of companions with the Monte Carlo method. As a result, we identified nearly two dozen of eclipsing systems that might have companions with a minimum mass larger than that of a neutron star. Their masses fall into the range between 1.7 and 34 solar masses. This sample of triples with high mass companions can be confirmed with the help of observations gathered by Gaia: parallaxes and astrometric measurements.
\end{abstract}

Keywords. stars: distances, binaries: eclipsing

\section{Sample selection}

This work is aimed at discovering massive companions, and we assumed the threshold at the mass of a neutron star. For this purpose, we examined the $\mathrm{O}-\mathrm{C}$ diagrams created from minima timings collected by Kreiner (2004), and based on their shape, exhibiting significant changes and possible cyclic variations as well as large amplitude, we selected 79 binaries out of more than a thousand. For these, assuming that the changes are being due to companions, we made preliminary computations and derived the orbits of third bodies. The sample of interest of this work was limited to these binaries which meet the minimal mass of 1.7 solar masses (for details see: Jableka et al., 2013).

\section{Monte Carlo computations}

The inverse problem requires up to nine parameters, some of them are correlated. We applied the Monte Carlo search method to find the best fit in the nine-dimension space and allows to fit all nine, non orthogonal parameters simultaneously. We did the errors estimation by the jackknife re-sampling technique. For all systems we adjusted the linear ephemerides and a periodical LTE. In most cases we also fitted the quadratic term in the ephemerides.

No quadratic terms in the ephemerides of Y Cam, SZ Cam, RX Gem, T LMi and RW Per were needed. For these systems a periodical term is clearly noticeable. For U Cep, TU Her, CC Her, SW Oph and Y Psc mixed quadratic and periodical terms solution were required. Three systems: V602 Aql, V442 Cas and BO Gem, have their O-C diagrams with poor coverage, as for those, only one minimum in the minima timings is visible. V602 Aql and BO Gem have visible inflection points on both sides of relatively well covered minima, what suggests a possibility of periodical solutions. The same concerns 


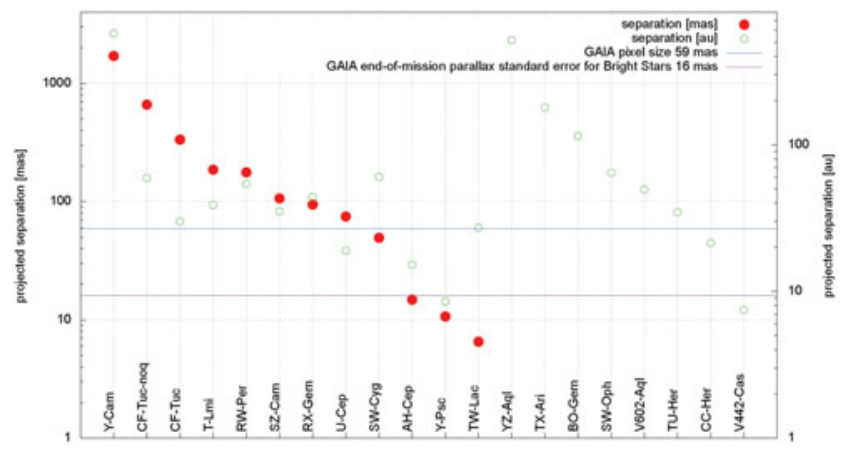

Figure 1. Projected separations of tertiaries to the center of mass of binary systems

YZ Aql and RX Gem in which the scatter in timings is comparable with the amplitude of the periodical solution. We obtained a very good fit for TW Lac and the residuals between observations and the periodic fit, may indicate an existence of another body, making this system to be a quadruple one.

\section{Summary}

Taking into account the total mass of the binary systems considered (taken from the literature), we calculated the third bodies mass functions, and, from these, their lower mass limits. For systems to which the distance is known, we also calculated the projected distances of the companions to the center of mass of the companion and the binary, treated as a single mass. The results are shown in Fig. 1. All hypothetical companions have wide or very wide orbits, orbiting the binaries in a distance from 6 to more than 160 AU. For the systems with known distances this translates to the projected distance (for inclination of 90 degrees) in the range between about 7 and 1100 mas.

For 18 systems we derived the minimum masses larger than than the threshold limit of $1.7 \mathrm{M}_{\odot}$. The most massive tertiary (about $34 \mathrm{M}_{\odot}$ ) was obtained for YZ Aql. Having such large masses, the companions should be detected in photometric and/or spectroscopic observations. In the case of these undetected, despite of their high mass, e.q. CF Tuc (Dogru et al., 2009), an improved distance determination and astrometric measurements made by Gaia can confirm their presence, and if not, a more detail investigation of magnetic cycles theories will be required.

The Applegate mechanism, often considered as an explanation of cyclic or pseudocyclic trends in the O-C diagrams of eclipsing binaries, could be applied to 4 systems in our sample (SZ Cam, V442 Cas, U Cep and Y Psc). The amplitude of variations is too large for remaining 14 binaries, and the Applegate mechanism can not be responsible for such large O-C variations.

\section{Aknowledgements}

This study was partly funded by the NCN 2012/05/E/ST9/03915 grant.

\section{References}

Dogru, D., Erdem, A., Dogru, S. S., \& Zola, S. 2009, MNRAS, 397, 1647

Jableka, D., Zola, S., Kreiner, J. M., \& Zakrzewski, B. 2013, CEAB, 37, 195

Kreiner, J. M. 2004, AcA, 54, 207 